\title{
GROUP-THEORETIC AXIOMS FOR PROJECTIVE GEOMETRY
}

\author{
Alex D. Gottlieb and Joseph Lipman
}

\begin{abstract}
We show that a certain category $\mathbb{G}$, whose objects are pairs $G \supset H$ of groups subject to simple axioms, is equivalent to the category of $\geq 2$-dimensional vector spaces and injective semi-linear maps; and deduce via the "Fundamental Theorem of Projective Geometry" that the category of $\geq 2$-dimensional projective spaces is equivalent to the quotient of a suitable subcategory of $\mathbb{G}$ by the least equivalence relation which identifies conjugation by any element of $H$ with the identity automorphism of $G$.
\end{abstract}

Introduction. Let $V$ be a left vector space of dimension $\geq 2$ over a (not necessarily commutative) field $F$. For any pair $0 \neq a \in F, v \in V$, let $[a, v]: V \rightarrow V$ be the map given by

$$
[a, v](w)=a w+v \quad(w \in V) .
$$

The set $G$ of all such maps is closed under composition:

$$
[a, v] \circ\left[a^{\prime}, v^{\prime}\right]=\left[a a^{\prime}, a v^{\prime}+v\right] ;
$$

and each $[a, v]$ is bijective, with inverse

$$
[a, v]^{-1}=\left[a^{-1},-a^{-1} v\right] .
$$

So $G$ is a group of transformations of $V$, with identity element $e=[1,0]$.

Denote by $H$ the subgroup of $G$ consisting of all maps of the form $[a, 0] . H$ is isomorphic to the multiplicative group of non-zero elements in $F$.

Denote by $T$ the subgroup of $G$ consisting of all maps of the form $[1, v] . T$ is a normal subgroup of $G$, isomorphic to the additive group $V$. Every element of $G$ is uniquely of the form $t h$, with $t \in T, h \in H$ :

$$
[a, v]=[1, v][a, 0] .
$$

The elements of $T$ are called translations. One checks that:

(0.1) A non-identity element $g \in G$ is a translation if and only if no conjugate of $g$ lies in $H$.

Recall that for $g \in G$, the double coset $H g H$ is the set

$$
H g H=\left\{h_{1} g h_{2} \mid g \in G \text { and } h_{1}, h_{2} \in H\right\} .
$$

1991 Mathematics Subject Classification. 15A99, 20F29, 51A05.

Key words and phrases. groups, semi-linear maps, projective geometry, equivalence of categories.

Supported through NSF contract DMS-8803054 at Purdue University, the first author by a Research Experiences for Undergraduates supplement. 
One verifies the following properties of the pair $(G, H)$.

(GH1) For all $g \in G$,

$$
G \neq H g H \cup H .
$$

(In other words $H$ has at least three distinct double cosets in $G$.)

(GH2) For all $g \in G$,

$$
g H g^{-1} \subset H g H \cup\{e\} .
$$

(GH3) For all $g \in G$,

$$
H g H=H g^{-1} H \text {. }
$$

Remarks. (i) With regard to (GH2), note that for $g \in G$ and $h \in H$,

$$
g h g^{-1}=e \Longleftrightarrow h=e .
$$

(ii) The property (GH3) follows formally from (GH2), except when $H=\{e\}$, in which case (GH2) says nothing at all.

Indeed, if $g \in G$ and $e \neq h \in H$, then applying (GH2) twice we get

$$
\begin{gathered}
g h^{-1} g^{-1} \in H g H, \\
g h^{-1} g^{-1}=\left(g h g^{-1}\right)^{-1} \in(H g H)^{-1}=H g^{-1} H ;
\end{gathered}
$$

so the double cosets $H g H$ and $H g^{-1} H$ meet, and hence they are equal.

(iii) We will see later (Remark 1.11) that given (GH1), conditions (GH2) and (GH3) together are group-theoretically equivalent to :

$(\mathrm{GH} 3)^{*} \quad$ for each $g \in G, H g H \cup H$ is a subgroup of $G$.

Now let $\mathbb{V}$ be the category whose objects are all pairs $(V, F)$ as above, and whose arrows are injective semi-linear maps

$$
(\zeta, \theta):(V, F) \rightarrow\left(V^{\prime}, F^{\prime}\right)
$$

More specifically:

- $\theta: F \rightarrow F^{\prime}$ is a homomorphism of fields.

$-\zeta: V \rightarrow V^{\prime}$ is an injective map satisfying

$$
\begin{aligned}
\zeta\left(v_{1}+v_{2}\right) & =\zeta\left(v_{1}\right)+\zeta\left(v_{2}\right) & & \left(v_{1}, v_{2} \in V\right), \\
\zeta(a v) & =\theta(a) \zeta(v) & & (a \in F, v \in V) .
\end{aligned}
$$


Let $\mathbb{G}$ be the category whose objects are pairs $(G, H)$ consisting of a group $G$ and a subgroup $H$ satisfying (GH1)-(GH3) above, and whose arrows $(G, H) \rightarrow\left(G^{\prime}, H^{\prime}\right)$ are those group homomorphisms $f: G \rightarrow G^{\prime}$ satisfying

$(0.2 .1) f(H) \subset\left(H^{\prime}\right)$, and

(0.2.2) if $g \in G$ has no conjugate lying in $H$, then $f(g) \in G^{\prime}$ has no conjugate lying in $H^{\prime}$.

We define a functor $\Gamma: \mathbb{V} \rightarrow \mathbb{G}$ as follows:

- For any object $(V, F), \Gamma(V, F)$ is the pair $(G, H)$ described at the beginning of this Introduction.

- For any arrow $(\zeta, \theta):(V, F) \rightarrow\left(V^{\prime}, F^{\prime}\right)$, the arrow

$$
\Gamma(\zeta, \theta): \Gamma(V, F) \rightarrow \Gamma\left(V^{\prime}, F^{\prime}\right)
$$

is given by the formula

$$
\Gamma(\zeta, \theta)[a, v]=[\theta(a), \zeta(v)] .
$$

To verify that $\Gamma$ is indeed a functor, check that it respects categorical identities and composition, and that $\Gamma(\zeta, \theta)$ is a group homomorphism satisfying (0.2.1) and (0.2.2) - cf. (0.1).

Our main result is:

Theorem 1. $\Gamma$ is an equivalence of categories.

In other words, there exists a functor $\Theta: \mathbb{G} \rightarrow \mathbb{V}$ together with isomorphisms of functors

$$
\Gamma \Theta \stackrel{\sim}{\longrightarrow} \mathbf{1}_{\mathbb{G}}, \quad \Theta \Gamma \stackrel{\sim}{\longrightarrow} \mathbf{1}_{\mathbb{V}}
$$

(where 1 denotes an identity functor). Such a functor $\Theta$ is called a pseudo-inverse of $\Gamma$.

The title of this paper refers to Theorem 2 below. By way of explanation, we first reformulate the Fundamental Theorem of Projective Geometry [A, p. 88] in the language of categories.

The projective space $P=P(V, F)$ is, by definition, the set of one-dimensional subspaces of $V$. A projective subspace of $P$ is a subset consisting of all the one-dimensional subspaces of some vector subspace of $V$.

A sequence $\left(x_{1}, x_{2}, \ldots, x_{n}\right)$ in $P$ is linearly independent if for each $i=1,2, \ldots, n, x_{i}$ lies outside some projective subspace $P_{i} \subset P$ which contains $x_{j}$ for all $j \neq i ;^{1}$ and linearly dependent otherwise. Three points $x_{1}, x_{2}, x_{3} \in P$ are collinear if $\left(x_{1}, x_{2}, x_{3}\right)$ is linearly dependent.

A map

$$
\pi: P(V, F)=P \rightarrow P^{\prime}=P\left(V^{\prime}, F^{\prime}\right)
$$

is a collineation if for any three collinear points $x_{1}, x_{2}, x_{3} \in P$ the points $\pi\left(x_{1}\right), \pi\left(x_{2}\right), \pi\left(x_{3}\right) \in$ $P^{\prime}$ are also collinear. A rather simple induction shows that this is equivalent to $\pi$ mapping every linearly dependent sequence in $P$ to a linearly dependent sequence in $P^{\prime}$. We say that $\pi$ is linearly faithful if it satisfies the following conditions, which are (exercise) equivalent:

- A sequence $\left(x_{1}, x_{2}, \ldots, x_{n}\right)$ in $P$ is linearly independent if and only if the sequence $\left(\pi\left(x_{1}\right), \pi\left(x_{2}\right), \ldots, \pi\left(x_{n}\right)\right)$ in $P^{\prime}$ is linearly independent.

- Every projective subspace $P_{1} \subset P$ is of the form $\pi^{-1}\left(P_{1}^{\prime}\right)$ for some projective subspace $P_{1}^{\prime} \subset P^{\prime}$.

\footnotetext{
${ }^{1}$ i.e., if the subspace $x_{1}+x_{2}+\cdots+x_{n} \subset V$ spanned by the $x_{i}$ has dimension $n$.
} 
Any linearly faithful map is an injective collineation. A bijective collineation $\pi$ is linearly faithful if and only if $\pi^{-1}$ is a collineation.

We define $\mathbb{P}_{2}$, the category of projective spaces of dimension $\geq 2$, as follows:

- The objects of $\mathbb{P}_{2}$ are the vector spaces $(V, F)$ of dimension $\geq 3$.

- The arrows $(V, F) \rightarrow\left(V^{\prime}, F^{\prime}\right)$ in $\mathbb{P}_{2}$ are the linearly faithful maps $\pi: P(V, F) \rightarrow$ $P\left(V^{\prime}, F^{\prime}\right)$.

Let $\mathbb{V}_{3}$ be the subcategory of $\mathbb{V}$ with objects the vector spaces $(V, F)$ of dimension $\geq 3$, and with arrows the semi-linear maps

$$
(\zeta, \theta):(V, F) \rightarrow\left(V^{\prime}, F^{\prime}\right)
$$

such that $\zeta$ is linearly faithful in the sense that it satisfies the following equivalent conditions:

- A sequence $\left(v_{1}, v_{2}, \ldots, v_{n}\right)$ in $V$ is linearly independent (over $F$ ) if and only if the sequence $\left(\zeta\left(x_{1}\right), \zeta\left(x_{2}\right), \ldots, \zeta\left(x_{n}\right)\right)$ in $V^{\prime}$ is linearly independent (over $\left.F^{\prime}\right)$.

- Every linear subspace $V_{1} \subset V$ is of the form $\zeta^{-1}\left(V_{1}^{\prime}\right)$ for some linear subspace $V_{1}^{\prime} \subset V^{\prime}$.

Remarks. (i) If $\zeta$ is linearly faithful then $\zeta$ is injective.

(ii) If $\zeta$ is injective and $\theta$ is bijective then $\zeta$ is linearly faithful. ${ }^{2}$

(iii) If $\operatorname{dim} V \geq 3$, and if $a$ is any non-zero element of $F$, then the automorphism $\left(\zeta_{a}, \theta_{a}\right):(V, F) \rightarrow(V, F)$ given by

$$
\begin{aligned}
& \zeta_{a}(v)=a v \quad(v \in V) \\
& \theta_{a}(b)=a b a^{-1} \quad(b \in F)
\end{aligned}
$$

is an arrow in $\mathbb{V}_{3}$.

We define a functor $\Pi: \mathbb{V}_{3} \rightarrow \mathbb{P}_{2}$ by

$$
\begin{aligned}
& \Pi(V, F)=(V, F), \\
& \Pi\left((\zeta, \theta):(V, F) \rightarrow\left(V^{\prime}, F^{\prime}\right)\right)=\pi_{\zeta}
\end{aligned}
$$

where for each non-zero $v \in V, \pi_{\zeta}$ takes the subspace $F v \in P(V, F)$ to the subspace $F^{\prime} \zeta(v) \in$ $P\left(V^{\prime}, F^{\prime}\right)$.

Next we form a quotient category of $\mathbb{V}_{3}$ through which $\Pi$ factors. Let $\mathbf{R}$ be the equivalence relation under which two arrows in $V_{3}$

$$
\left(\zeta_{1}, \theta_{1}\right),\left(\zeta_{2}, \theta_{2}\right):(V, F) \rightarrow\left(V^{\prime}, F^{\prime}\right)
$$

are equivalent if there exists a non-zero $a \in F^{\prime}$ such that

$$
\left(\zeta_{2}, \theta_{2}\right)=\left(\zeta_{a}, \theta_{a}\right) \circ\left(\zeta_{1}, \theta_{1}\right)
$$

The quotient category $\mathbb{V}_{3} / \mathbf{R}$ has the same objects as $\mathbb{V}_{3} ;$ but for two objects $(V, F)$, $\left(V^{\prime}, F^{\prime}\right)$, the $\mathbb{V}_{3} / \mathbf{R}$-arrows $(V, F) \rightarrow\left(V^{\prime}, F^{\prime}\right)$ are the equivalence classes under $\mathbf{R}$ of arrows in $\mathbb{V}_{3}$ between these two objects, composition being defined in the natural way.

There is a canonical functor $\rho: \mathbb{V}_{3} \rightarrow \mathbb{V}_{3} / \mathbf{R}$ taking any object to itself and taking any arrow to its equivalence class; and it is easily checked that there is a unique functor

$$
\bar{\Pi}: \mathbb{V}_{3} / \mathbf{R} \longrightarrow \mathbb{P}_{2}
$$

such that

$$
\Pi=\bar{\Pi} \circ \rho .
$$

Now, at last, we can state:

\footnotetext{
${ }^{2}$ More generally, $\zeta$ is linearly faithful $\Longleftrightarrow$ the map $F^{\prime} \otimes_{F} V \rightarrow V^{\prime}$ induced by $(\zeta, \theta)$ is injective.
} 
Fundamental Theorem of Projective Geometry. The functor $\bar{\Pi}$ is an isomorphism of categories.

In other words, $\bar{\Pi}$ is bijective on objects (clearly) and on arrows: every arrow in $\mathbb{P}_{2}$ is of the form $\pi_{\zeta}$, and

$$
\pi_{\zeta}=\pi_{\zeta^{\prime}} \Longleftrightarrow \zeta \equiv \zeta^{\prime} \quad(\bmod \mathbf{R}) .
$$

The proof is essentially given by E. Artin in [A, pp. 88-91]. Artin restricts his attention to finite-dimensional spaces and to arrows which are isomorphisms; but his arguments are easily modified to cover the present statement.

The Fundamental Theorem in some sense reduces Projective Geometry to Linear Algebra. Consequently, using Theorem 1, we can reduce Projective Geometry to Group Theory. Here is a precise formulation; proofs are provided in $\S 2$.

Let $\mathbb{G}_{3}$ be the subcategory of $\mathbb{G}$ whose objects are pairs $(G, H)$ satisfying $(\mathrm{GH} 2)$, (GH3)or $(\mathrm{GH} 2)^{*},(\mathrm{GH} 3)^{*}$ - and $(\mathrm{GH} 1)^{*}$ For all $g_{1}, g_{2} \in G$,

$$
G \neq\left(H g_{1} \cup H\right)\left(H g_{2} \cup H\right) ;
$$

and whose arrows $f:(G, H) \rightarrow\left(G^{\prime}, H^{\prime}\right)$ are those of $\mathbb{G}$ which further satisfy:

(0.3) Every subgroup $G_{1}$ of $G$ containing $H$ is of the form $f^{-1}\left(G_{1}^{\prime}\right)$

for some subgroup $G_{1}^{\prime}$ of $G^{\prime}$ containing $H^{\prime}$.

If $\Theta$ is, as above, a pseudo-inverse of $\Gamma$, then (GH1)* is equivalent to the vector space $\Theta(G, H)$ having dimension $\geq 3$ (cf. Corollary $(2.2)$, which also shows that if $(*)$ holds for some two elements $g_{1}, g_{2} \in G$ such that

$$
H \neq H g_{1} H \neq H g_{2} H \neq H
$$

then it holds for all $\left.g_{1}, g_{2} \in G\right)$; and (0.3) is equivalent to $\Theta(f)$ being linearly faithful. In fact the above functors $\Gamma$ and $\Theta$ induce pseudo-inverse equivalences between the categories $\mathbb{V}_{3} \subset \mathbb{V}$ and $\mathbb{G}_{3} \subset \mathbb{G}$.

Note that if $(G, H) \in \mathbb{G}_{3}$ then for any $h \in H$, the inner automorphism $\gamma_{h}: G \rightarrow G$, given by

$$
\gamma_{h}(g)=h g h^{-1} \quad(g \in G)
$$

is an arrow in $\mathbb{G}_{3}$.

On $\mathbb{G}_{3}$ we consider the equivalence relation $\mathbf{R}^{*}$ under which two arrows

$$
f_{1}, f_{2}:(G, H) \rightarrow\left(G^{\prime}, H^{\prime}\right)
$$

are equivalent if there exists an $h \in H^{\prime}$ such that $f_{2}=\gamma_{h} \circ f_{1}$. As above, we have a quotient category $\mathbb{G}_{3} / \mathbf{R}^{*}$, together with a canonical functor $\rho^{*}: \mathbb{G}_{3} \rightarrow \mathbb{G}_{3} / \mathbf{R}^{*}$. And there is a unique functor $\bar{\Theta}: \mathbb{G}_{3} / \mathbf{R}^{*} \rightarrow \mathbb{V}_{3} / \mathbf{R}$ making the following diagram commute:

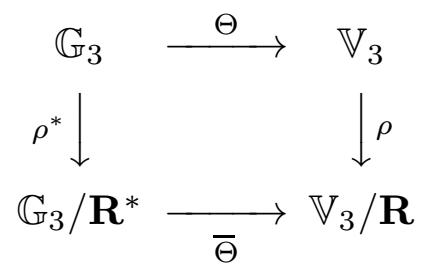

This $\bar{\Theta}$ is also an equivalence of categories.

Following $\bar{\Theta}$ by the isomorphism $\bar{\Pi}$, we obtain the above-indicated group-theoretic foundations for projective geometry: 
Theorem 2. The category $\mathbb{P}_{2}$ of projective spaces of dimension $\geq 2$ is equivalent to the quotient category $\mathbb{G}_{3} / \mathbf{R}^{*}$ just described.

1. Proof of Theorem 1. We first define a category $\mathbb{S}$ which will serve as an intermediary in the proof of the equivalence of $\mathbb{G}$ and $\mathbb{V}$.

The objects of $\mathbb{S}$, called "pointed geometries," are triples $(G, S, p)$ with $S$ a set, $p \in S$, and $G$ a group acting faithfully on $S$, i.e., there is a map $G \times S \rightarrow S$-for which the image of a pair $(g, s)$ is denoted $g s$ - such that

$$
(g h) s=g(h s) \quad(g, h \in G ; s \in S)
$$

and such that

$$
g s=s \text { for all } s \in S \Longleftrightarrow g=e \text {, the identity. }
$$

(For motivation, consider the triple $(G, V, 0)$ described at the beginning of the Introduction; or cf. [L]). A translation of $(G, S, p)$ is defined as in [L, p. 272] to be an element $g \in G$ such that either $g=e$ or $g$ has no fixed points (i.e., $g s \neq s$ for all $s \in S$ ). A line of $(G, S, p)$ is by definition a subset of $S$ of the form

$$
s_{1}+s_{2}=\left\{s \in S \mid s=s_{1} \text { or } \exists g \in G \text { with } g s_{1}=s_{1}, g s_{2}=s\right\}
$$

where $s_{1}, s_{2}$ are distinct points in $S$. It is assumed further that $G$ acts doubly transitively on lines, but not on all of $S$. (Cf. [L, p. 268, AXIOM 1, and p. 271, AXIOM 2]; recall that a group acts doubly transitively on a set if for any $s_{1}, s_{2}, s_{3}, s_{4}$ in the set with $s_{1} \neq s_{2}, s_{3} \neq s_{4}$, there is a $g$ in the group such that $g s_{1}=s_{3}$ and $g s_{2}=s_{4}$.) Note that then $G$ acts transitively on $S$, i.e., for any $s_{1}, s_{2}$ in $S$ there is a $g$ in $G$ with $g s_{1}=s_{2}$. (There is even such a $g$ which furthermore satisfies $g s_{2}=s_{1}$ : this is obvious if $s_{1}=s_{2}$, and otherwise holds because $s_{1}$ and $s_{2}$ both lie on the line $\left.s_{1}+s_{2}\right)$.

The arrows of $\mathbb{S}$ are pairs

$$
(\phi, \psi):(G, S, p) \rightarrow\left(G^{\prime}, S^{\prime}, p^{\prime}\right)
$$

where $\phi: G \rightarrow G^{\prime}$ is a group homomorphism and $\psi: S \rightarrow S^{\prime}$ is a map of sets with $\psi(p)=p^{\prime}$, such that

$$
\psi(g s)=\phi(g) \psi(s) \quad(g \in G, s \in S)
$$

and such that

$$
\begin{aligned}
& \phi \text { takes non-identity translations of }(G, S, p) \\
& \text { to non-identity translations of }\left(G^{\prime}, S^{\prime}, p^{\prime}\right) .
\end{aligned}
$$

Composition of arrows $(\phi, \psi):(G, S, p) \rightarrow\left(G^{\prime}, S^{\prime}, p^{\prime}\right)$ and $\left(\phi^{\prime}, \psi^{\prime}\right):\left(G^{\prime}, S^{\prime}, p^{\prime}\right) \rightarrow\left(G^{\prime \prime}, S^{\prime \prime}, p^{\prime \prime}\right)$ is defined in the obvious way:

$$
\left(\phi^{\prime}, \psi^{\prime}\right) \circ(\phi, \psi)=\left(\phi^{\prime} \circ \phi, \psi^{\prime} \circ \psi\right):(G, S, p) \rightarrow\left(G^{\prime \prime}, S^{\prime \prime}, p^{\prime \prime}\right) .
$$

We observe in passing the following facts.

Remarks (1.3). Let $(\phi, \psi)$ be a pair as above satisfying (1.1).

(1) If $\psi$ is bijective then (1.2) holds.

(2) If (1.2) holds then both $\phi$ and $\psi$ are injective. 
Proof. (1) Assuming $\psi$ to be bijective, let g be a non-identity translation of $(G, S, p)$, so that $g s \neq s$ for any $s \in S$. Since $\psi$ is injective, therefore

$$
\phi(g) \psi(s)=\psi(g s) \neq \psi(s)
$$

and since $\psi$ is surjective, this means that $\phi(g) s^{\prime} \neq s^{\prime}$ for any $s^{\prime} \in S^{\prime}$, i.e., $\phi(g)$ is a non-identity translation of $\left(G^{\prime}, S^{\prime}, p^{\prime}\right)$. Thus (1.2) holds.

(2) Assuming (1.2), let us show first that $\psi$ is injective. Let $s_{1}, s_{2}$ be distinct points of S. By [L, p. 272, Thm. 8] there exists a translation $g$ (obviously non-identity) with $g s_{1}=s_{2}$. Then

$$
\psi\left(s_{2}\right)=\psi\left(g s_{1}\right)=\phi(g) \psi\left(s_{1}\right) \neq \psi\left(s_{1}\right)
$$

because $\phi(g)$ is a non-identity translation. So $\psi$ is indeed injective.

Finally, if $g \in G$ is such that $\phi(g)=e^{\prime}$, the identity in $G^{\prime}$, then for every $s \in S$ we have

$$
\psi(g s)=\phi(g) \psi(s)=\psi(s),
$$

and since $\psi$ is injective, therefore $g s=s$. So $g=e$, and $\phi$ is injective, as asserted.

We show now that:

Theorem 1a. The categories $\mathbb{G}$ and $\mathbb{S}$ are equivalent.

Proof. The asserted equivalence is induced by a well-known equivalence

$$
\Phi: \overline{\mathbb{G}} \rightarrow \overline{\mathbb{S}}
$$

where the categories $\overline{\mathbb{G}} \supset \mathbb{G}$ and $\overline{\mathbb{S}} \supset \mathbb{S}$ are as follows:

The objects of $\overline{\mathbb{G}}$ are pairs $(G, H)$ with $G$ a group and $H$ a subgroup of $G$; and the arrows $(G, H) \rightarrow\left(G^{\prime}, H^{\prime}\right)$ are the group homomorphisms $f: G \rightarrow G^{\prime}$ for which $f(H) \subset H^{\prime}$.

The objects of $\overline{\mathbb{S}}$ are triples $(G, S, p)$ with $S$ a set, $p \in S$, and $G$ a group acting transitively on $S$; and the arrows $(G, S, p) \rightarrow\left(G^{\prime}, S^{\prime}, p^{\prime}\right)$ are pairs of maps $(\phi, \psi)$ as above, satisfying (1.1) (but not necessarily (1.2)).

For $(G, H) \in \bar{G}$, let $G / H$ be the set consisting of all the left cosets of $H$ in $G$. $G$ acts transitively on $G / H$ by left multiplication.

The above equivalence $\Phi$ is the functor given by

$$
\begin{aligned}
\Phi(G, H) & =(G, G / H, H) \\
\Phi\left(f:(G, H) \rightarrow\left(G^{\prime}, H^{\prime}\right)\right) & =\left(f, \psi_{f}\right):(G, G / H, H) \rightarrow\left(G^{\prime}, G^{\prime} / H^{\prime}, H^{\prime}\right)
\end{aligned}
$$

where

$$
\psi_{f}(g H)=f(g) H^{\prime} \quad(g \in G) .
$$

For $(G, S, p) \in \bar{S}$, let $G_{p} \subset G$ be the stabilizer of $p$, i.e., the subgroup

$$
G_{p}=\{g \in G \mid g p=p\} .
$$

It is straightforward to verify that a pseudo-inverse of $\Phi$ is the functor $\Psi: \overline{\mathbb{S}} \rightarrow \overline{\mathbb{G}}$ given by

$$
\begin{aligned}
\Psi(G, S, p) & =\left(G, G_{p}\right) \\
\Psi\left((\phi, \psi):(G, S, p) \rightarrow\left(G^{\prime}, S^{\prime}, p^{\prime}\right)\right) & =\phi:\left(G, G_{p}\right) \rightarrow\left(G^{\prime}, G_{p^{\prime}}^{\prime}\right)
\end{aligned}
$$


In fact $\Psi \Phi=\mathbf{1}_{\overline{\mathbb{G}}}$; and a functorial isomorphism $\Phi \Psi \stackrel{\sim}{\longrightarrow} \mathbf{1}_{\mathbb{S}}$ is given for $(G, S, p) \in \overline{\mathbb{S}}$ by

$$
(1, \psi): \Phi \Psi(G, S, p)=\left(G, G / G_{p}, G_{p}\right) \stackrel{\sim}{\longrightarrow}(G, S, p)
$$

where $\psi\left(g G_{p}\right)=g p$.

We will show that $\Phi$ maps the subcategory $\mathbb{G}$ of $\overline{\mathbb{G}}$ into the subcategory $\mathbb{S}$ of $\overline{\mathbb{S}}$, and that $\Psi$ maps $\mathbb{S}$ into $\mathbb{G}$. It is easily checked that an isomorphism in $\overline{\mathbb{G}}$ between two objects of $\mathbb{G}$ is actually an isomorphism in $\mathbb{G}$; and similarly for $\mathbb{S} \subset \overline{\mathbb{S}}$. It will follow then that the restrictions of $\Phi$ and $\Psi$ to the respective subcategories $\mathbb{G}, \mathbb{S}$ are pseudo-inverse equivalences, proving Theorem 1a.

From the simple fact that $g \in G$ is a non-identity translation of $(G, S, p) \in \overline{\mathbb{G}}$ (i.e., $g s \neq s$ for all $s \in S$ ) if and only if no conjugate of $g$ lies in $G_{p}$, it follows easily that $\Phi$ takes arrows of $\mathbb{G}$ to arrows of $\mathbb{S}$, and that $\Psi$ takes arrows of $\mathbb{S}$ to arrows of $\mathbb{G}$. So it remains to examine the effect of $\Phi$ (resp. $\Psi$ ) on objects of $\mathbb{G}$ (resp. $\mathbb{S})$.

Let us show that:

$$
\Phi(G, H)=(G, G / H, H) \in \mathbb{S} \quad \text { for }(G, H) \in \mathbb{G} .
$$

(1.4.1) $G$ acts faithfully on $G / H$.

We must show: if $g^{\prime} \in G$ is such that $g^{\prime} g H=g H$ for all $g H \in G / H$, i.e., $g^{\prime} \in g H g^{-1}$ for all $g \in G$, then $g^{\prime}=e$. But by (GH1) there is a $g \in G$ such that the double cosets $H g H$ and $H$ are distinct, whence $H g H \cap H=\emptyset$; and from (GH2) we then get

$$
g^{\prime} \in g H g^{-1} \cap e H e^{-1} \subset(H g H \cup\{e\}) \cap H=\{e\} .
$$

(1.4.2) $G$ does not act doubly transitively on $G / H$.

By (GH1) there exist three distinct double cosets $H, H g H, H g^{\prime} H$. Then $H \neq g^{-1} H$ and $g^{\prime} H \neq H$, but there is no $j \in G$ such that $j H=g^{\prime} H$ and $j g^{-1} H=H$, since such a $j$ would lie in $H g^{\prime} H \cap H g H=\emptyset$.

(1.4.3) $G$ acts doubly transitively on lines.

We first give a condition for $c H \in G / H$ to lie on a line $a H+b H$.

Lemma(1.5). If $a H \neq b H$ and $a H \neq c H$ are in $G / H$, then

$$
\begin{aligned}
& c H \in a H+b H \underset{(\mathrm{i})}{\Longleftrightarrow} a^{-1} c \in H a^{-1} b H \\
& \underset{\text { (ii) }}{\Longleftrightarrow}(a H)^{-1}(c H)=(a H)^{-1}(b H) \text {. }
\end{aligned}
$$

Proof. Since $c H \neq a H$, we have

$$
\begin{aligned}
c H \in a H+b H & \Longleftrightarrow \exists g \in G \text { with } g a H=a H, g b H=c H \\
& \Longleftrightarrow a H a^{-1} \cap c H b^{-1} \neq \emptyset \\
& \Longleftrightarrow H a^{-1} b \cap a^{-1} c H \neq \emptyset \\
& \Longleftrightarrow a^{-1} c \in H a^{-1} b H .
\end{aligned}
$$


This proves the logical equivalence (i); and (ii) is obvious.

Now let $g_{1} H, g_{2} H, g_{3} H, g_{4} H \in a H+b H, g_{1} H \neq g_{2} H, g_{3} H \neq g_{4} H$. By (1.5),

$$
a^{-1} g_{i} \in H \cup H a^{-1} b H \quad(i=1,2,3,4) .
$$

Note that $a^{-1} g_{i} \in H \Longleftrightarrow g_{i} H=a H$. In particular, $a^{-1} g_{1}$ and $a^{-1} g_{2}$ can't both lie in $H$. It follows that

$$
g_{1}^{-1} g_{2}=\left(g_{1}^{-1} a\right)\left(a^{-1} g_{2}\right) \in H b^{-1} a H \cup H a^{-1} b H \cup\left(H b^{-1} a H\right)\left(H a^{-1} b H\right) .
$$

By (GH3),

$$
H b^{-1} a H=H a^{-1} b H
$$

and by (GH2),

$$
\left(H b^{-1} a H\right)\left(H a^{-1} b H\right)=H\left(b^{-1} a H a^{-1} b\right) H \subset H b^{-1} a H \cup H ;
$$

since $g_{1} H \neq g_{2} H$, therefore $g_{1}^{-1} g_{2} \notin H$, and we conclude then that $g_{1}^{-1} g_{2} \in H a^{-1} b H$.

Similarly, $g_{3}^{-1} g_{4} \in H a^{-1} b H$. So

$$
H g_{1}^{-1} g_{2} H=H a^{-1} b H=H g_{3}^{-1} g_{4} H,
$$

i.e., there exist $h_{1}, h_{2} \in H$ such that

$$
h_{1} g_{1}^{-1} g_{2} h_{2}=g_{3}^{-1} g_{4}
$$

Thus, for $g_{1} H, g_{2} H, g_{3} H, g_{4} H$ on one line, $g_{1} H \neq g_{2} H, g_{3} H \neq g_{4} H$, there is an element of $G$, (namely $g_{3} h_{1} g_{1}^{-1}=g_{4} h_{2}^{-1} g_{2}^{-1}$ ) which sends $g_{1} H$ to $g_{3} H$ and $g_{2} H$ to $g_{4} H$.

This completes the proof of (1.4)

Let us show finally that

$$
\Psi(G, S, p)=\left(G, G_{p}\right) \in \mathbb{G} \quad \text { for }(G, S, p) \in \mathbb{S} .
$$

For convenience, set $G_{p}=H$.

$$
G \neq H g H \cup H \text { for any } g \in G \text {. }
$$

For, were $G=H g H \cup H$, then for any point $p \neq p_{1} \in S$ we would have (by transitivity, see the definition of $\mathbb{S}$ ), for some $g_{1} \in G$,

$$
p_{1}=g_{1} p=h_{1} g p \quad\left(h_{1} \in H\right),
$$

and since $h_{1} p=p$ therefore $p_{1} \in p+g p$. Thus the line $p+g p$ would be all of $S$; and since $G$ acts doubly transitively on lines, but not on all of $S$, we would have a contradiction.

(1.6.2) $H g H=H g^{-1} H$ for any $g \in G$.

We may assume that $g \notin H$, i.e., $g p \neq p$. Since $G$ acts doubly transitively on the line $p+g p$, there exists $g_{1} \in G$ such that

$$
g_{1} p=g p, \quad g_{1} g p=p .
$$

It follows that there exist $h_{1}, h_{2} \in H$ such that

$$
g_{1}=g h_{1}=h_{2} g^{-1} .
$$

Hence $g \in H g^{-1} H$, i.e., $H g H=H g^{-1} H$. 
(1.6.3) $g \mathrm{Hg}^{-1} \subset H g H \cup\{e\}$ for any $g \in G$.

We may assume that $g \notin H$. Let $h \in H$, with $h \notin g^{-1} H g$. Then $p, g^{-1} p$, and $h g^{-1} p$ are three distinct points on the line $L=p+g^{-1} p$. Since $G$ acts doubly transitively on $L$, there is a $j \in G$ such that

$$
j p=g^{-1} p \quad \text { and } \quad j g^{-1} p=h g^{-1} p .
$$

Then we have $j=g^{-1} h_{1}$ for some $h_{1} \in H$, and $g^{-1} h_{1} g^{-1} p=h g^{-1} p$, so that $h g^{-1} \in$ $g^{-1} h_{1} g^{-1} H$, whence

$$
g h g^{-1} \in H g^{-1} H \stackrel{(1.6 .2)}{=} H g H .
$$

It remains to consider elements of the form $g h g^{-1}$ with $h \in H \cap g^{-1} H g$. Such an $h$ has the two distinct points $p, g^{-1} p \in S$ as fixed points, and so by [L, p. 271, Thm. 5], $h=e$, and $g h g^{-1}=e$.

This completes the proof of (1.6), and of Theorem 1a.

Our next task is to describe a functor $\Theta: \mathbb{G} \rightarrow \mathbb{V}$ pseudo-inverse to $\Gamma$. This is basically an elaboration of $[\mathrm{L}, \S 7]$.

For $(G, H) \in \mathbb{G}$, set

$$
\begin{aligned}
T=T_{G, H} & =\{\text { translations of }(G, H)\} \\
& \stackrel{\text { def }}{=}\{e\} \cup\{g \in G \mid \text { no conjugate of } g \text { lies in } H\} .
\end{aligned}
$$

The set $T$ is closed under conjugation:

$$
j T j^{-1}=T \quad(j \in G) .
$$

As above, $\Phi(G, H)=(G, G / H, H) \in \mathbb{S}$; and it is immediate that $T$ is the set of all translations of $(G, G / H, H)$ - cf. beginning of this $\S 1$. Hence, by [L, p. 274, Thm.11], $T$ is an abelian (normal) subgroup of $G$. We also note, for later use, the following consequence of [L, p. 272, Thm. 8]:

(1.7) Each left coset $g H$ contains exactly one translation.

Let $E$ be the ring of additive endomorphisms of $T$, with zero-element $\mathbf{0}=\mathbf{0}_{E}$. We define a map $\gamma: H \rightarrow E$ by

$$
[\gamma(h)](t)=h t h^{-1} \quad(h \in H, t \in T) .
$$

According to [L, p.276], $\gamma$ is injective and $F=\gamma(H) \cup\{\mathbf{0}\}$ is a subfield of $E$. Thus $T$ is a vector space over $F$.

Extend $\gamma$ to $\widetilde{\gamma}: H \cup\{\mathbf{0}\} \rightarrow E$ sending $\mathbf{0}$ to $\mathbf{0}$. Since $\widetilde{\gamma}$ is injective, there is a unique field structure on $\widetilde{H}=H \cup\{\mathbf{0}\}$ such that $\widetilde{\gamma}$ is a field isomorphism. For this structure, the product of two elements $h_{1}, h_{2} \in H$ is the same as their product in $G$. In particular, the multiplicative identity $1_{\widetilde{H}}$ is $e$. The additive inverse $-h_{1} \in \widetilde{H}$ is the unique $h \in H$ such that

$$
h t h^{-1}=\left(h_{1} t h_{1}^{-1}\right)^{-1}=h_{1} t^{-1} h^{-1} \quad \text { for all } \quad t \in T
$$

If $h_{2} \neq-h_{1}$, then the sum $h_{1}+h_{2} \in \widetilde{H}$ is the unique $h \in H$ such that for all $t \in T$

$$
h t h^{-1}=h_{1} t h_{1}^{-1} h_{2} t h_{2}^{-1} .
$$


All this follows from [L, p. 276]. We leave it to the reader to explicate sums and products in $\widetilde{H}$ involving $\mathbf{0}$.

Accordingly, we can regard $T$ as a vector space over $\widetilde{H}$, with scalar multiplication

$$
h \cdot t=h t h^{-1} \quad(h \in H, t \in T) .
$$

Remark (1.9). Given $h_{1}, h_{2} \in H$, with $h_{1} \neq-h_{2}$, if $h \in H$ is such that (1.8) holds for one non-identity $t \in T$, then $h=h_{1}+h_{2}$.

This follows from [L, p. 275, Cor. 12.1] and the injectivity of $\gamma$.

The above-mentioned functor $\Theta$ is specified by:

$$
\begin{aligned}
\Theta(G, H) & =\left(T_{G, H}, \widetilde{H}\right) \\
\Theta\left(f:(G, H) \rightarrow\left(G^{\prime}, H^{\prime}\right)\right) & =\left(\zeta_{f}, \theta_{f}\right):\left(T_{G, H}, \widetilde{H}\right) \rightarrow\left(T_{G^{\prime}, H^{\prime}}, \widetilde{H^{\prime}}\right)
\end{aligned}
$$

where

$$
\begin{array}{rlrl}
\zeta_{f}(t) & =f(t) & \left(t \in T_{G, H}\right) \\
\theta_{f}(h) & =f(h) \quad(h \in H) \\
\theta_{f}(\mathbf{0}) & =\mathbf{0}^{\prime} . & &
\end{array}
$$

To see that $\Theta$ is indeed a functor from $\mathbb{G}$ to $\mathbb{V}$ we need to prove that $\left(\zeta_{f}, \theta_{f}\right)$ is an injective semi-linear map.

The injectivity of $\zeta_{f}$ follows from that of $f$, which in turn follows from the fact that $\Phi(f)=\left(f, \psi_{f}\right)$ is a map in $\mathbb{S}$ (cf. (2) in (1.3) above.)

The conditions

$$
\begin{aligned}
\zeta_{f}\left(t_{1}+t_{2}\right) & =\zeta_{f}\left(t_{1}\right)+\zeta_{f}\left(t_{2}\right) & & \left(t_{1}, t_{2} \in T_{G, H}\right) \\
\zeta_{f}(h t) & =\theta_{f}(h) \zeta_{f}(t) & & (h \in \widetilde{H}, t \in T) \\
\theta_{f}\left(h_{1} h_{2}\right) & =\theta_{f}\left(h_{1}\right) \theta_{f}\left(h_{2}\right) & & \left(h_{1}, h_{2} \in \widetilde{H}\right) \\
\theta_{f}\left(1_{\widetilde{H}}\right) & =1_{\widetilde{H^{\prime}}} & &
\end{aligned}
$$

are trivial to verify. It remains then to show that

$$
\theta_{f}\left(h_{1}+h_{2}\right)=\theta_{f}\left(h_{1}\right)+\theta_{f}\left(h_{2}\right) \quad\left(h_{1}, h_{2} \in \widetilde{H}\right) .
$$

If any one of $h_{1}, h_{2}$, or $h_{1}+h_{2}$ is $\mathbf{0}$, then (1.10) is obvious. Otherwise, we can apply $f$ to (1.8), where $h=h_{1}+h_{2}$ and $t$ is a non-identity translation (which exists by (1.7), since $G \neq H$, cf. (GH1)) to get

$$
f(h) f(t) f(h)^{-1}=f\left(h_{1}\right) f(t) f\left(h_{1}\right)^{-1} f\left(h_{2}\right) f(t) f\left(h_{2}\right)^{-1} .
$$

By (0.2.2), no conjugate of $f(t)$ lies in $H^{\prime}$, i.e., $f(t)$ is a non-identity translation of $\left(G^{\prime}, H^{\prime}\right)$. Hence by (1.9),

$$
f\left(h_{1}+h_{2}\right)=f(h)=f\left(h_{1}\right)+f\left(h_{2}\right),
$$

proving (1.10). 
Now that we have the functor $\Theta$, let us show that it is a pseudo-inverse of $\Gamma$.

For $(G, H) \in \mathbb{G}$, and $T=T_{G, H}$, definitions yield

$$
\Gamma \Theta(G, H)=(\{[h, t]\},\{[h, 0]\}) \quad(h \in H, t \in T)
$$

where $[h, t]: T \rightarrow T$ is the map defined by

$$
[h, t](\tau)=(\gamma(h) \tau) t=h \tau h^{-1} t \quad(\tau \in T) .
$$

We define a map

$$
\alpha=\alpha_{G, H}: \Gamma \Theta(G, H) \rightarrow(G, H)
$$

by

$$
\alpha[h, t]=t h .
$$

Clearly $\alpha\{[h, 0]\}=H$; and one checks (using the fact that $T$ is an abelian normal subgroup of $G$ ) that

$$
\alpha\left([h, t] \circ\left[h^{\prime}, t^{\prime}\right]\right)=t h t^{\prime} h^{\prime}=\alpha[h, t] \alpha\left[h^{\prime}, t^{\prime}\right],
$$

so that $\alpha$ is a map in the category $\overline{\mathbb{G}}$. Furthermore, $\alpha$ is an isomorphism in $\overline{\mathbb{G}}$ (hence, as previously remarked, in $\mathbb{G}$ ) because, by (1.7), every element in $G$ is uniquely of the form $t h .^{3}$ Finally, $\alpha$ is functorial, i.e., for any arrow $f:(G, H) \rightarrow\left(G^{\prime}, H^{\prime}\right)$ in $\mathbb{G}$, the resulting diagram

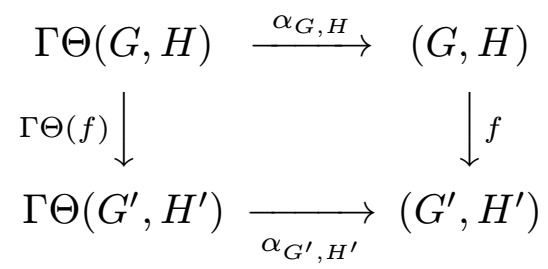

commutes, as follows easily from definitions. Thus we have an isomorphism of functors $\Gamma \Theta \stackrel{\sim}{\longrightarrow} \mathbf{1}_{\mathbb{G}}$.

Next, for $(V, F) \in \mathbb{V}$, let $(G, H)=\Gamma(V, F)$ be as in the Introduction, and let $T$ be the subgroup of $G$ consisting of all the translations $[1, v]$ with $v \in V$, so that with $\widetilde{H}$ as above we have

$$
\Theta \Gamma(V, F)=(T, \widetilde{H}) .
$$

We define a map

$$
(\zeta, \theta)=\left(\zeta_{V, F}, \theta_{V, F}\right): \Theta \Gamma(V, F) \rightarrow(V, F)
$$

by

$$
\begin{aligned}
\zeta[1, v] & =v & & (v \in V) \\
\theta[a, 0] & =a & & (0 \neq a \in F) \\
\theta(\mathbf{0}) & =0_{F} . & &
\end{aligned}
$$

\footnotetext{
${ }^{3}$ The reader who so desires can rephrase this argument in terms of semi-direct products.
} 
Both $\zeta$ and $\theta$ are bijective. We leave it to the reader to check (mechanically, via definitions) that $(\zeta, \theta)$ is semi-linear, and hence is an isomorphism in $\mathbb{V}$. For functoriality, we need to check that for any arrow $(\xi, \eta):(V, F) \rightarrow\left(V^{\prime}, F^{\prime}\right)$ in $\mathbb{V}$, the following diagram commutes:

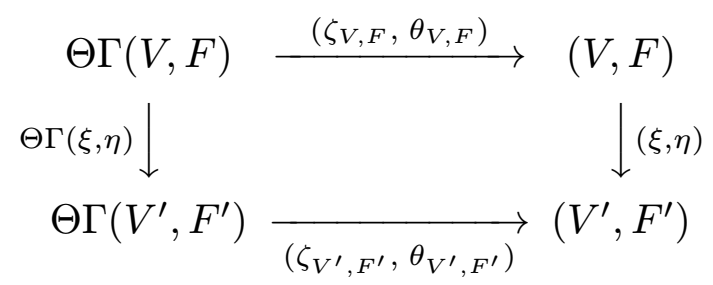

This again is a mechanical exercise. So we have an isomorphism of functors $\Theta \Gamma \stackrel{\sim}{\longrightarrow} \mathbf{1}_{\mathbb{V}}$; and $\Theta$ is indeed a pseudo-inverse of $\Gamma$.

This completes the proof of Theorem 1 .

Remark (1.11). Let us verify Remark (iii) in the Introduction. Assuming (GH1)-(GH3), we can prove (GH2)* as in (1.4.1); and we find that

$$
\begin{aligned}
(H g H \cup H)(H g H \cup H)^{-1} & =(H g H \cup H)\left(H g^{-1} H \cup H\right) \\
& =H g H g^{-1} H \cup H g H \cup H g^{-1} H \cup H \\
& \subset H g H \cup H,
\end{aligned}
$$

yielding (GH3)*. Conversely, (GH3)* easily implies (GH3) and that for any $g \in G$,

$$
g H g^{-1} \subset(H g H \cup H)(H g H \cup H)^{-1} \subset(H g H \cup H) .
$$

So to prove (GH2), we need to show that if $g \notin H$ then

$$
g H g^{-1} \cap H=\{e\} .
$$

For this, observe that (GH3)* alone is enough for the proof of (1.4.3), (GH1) is enough for (1.4.2), and (GH2)* is enough for (1.4.1). So we can apply [L, p. 271, Thm.5] to any $h \in g H g^{-1} \cap H$ : since $h H=H$ and $h g H=g H$, therefore $h=e$.

Remark (1.12). Here is a variation on the theme of Theorem 1.

Recall the definition of "faithful group action" given near the beginning of $\S 1$. Let $H$ be a group acting faithfully on a group $T$, via automorphisms, i.e.,

$$
h\left(t_{1} \cdot t_{2}\right)=h t_{1} \cdot h t_{2} \quad\left(h \in H ; t_{1}, t_{2} \in T\right)
$$

where $\cdot$ is the group operation in $T$. The orbit $\langle t\rangle$ of $t \in T$ is the set

$$
\langle t\rangle=\{h t \mid h \in H\} .
$$

For example, the orbit of the identity element $e_{T}$ consists of $e_{T}$ alone. 
Theorem 1b. Let $H$ be a group acting faithfully via automorphisms on a group $T$. Then the following conditions (1) and (2) are equivalent:

(1) (i) There are at least three distinct orbits in $T$, and

(ii) for any $t \in T,\langle t\rangle \cup\left\{e_{T}\right\}$ is a subgroup of $T$.

(2) There is a vector space $V$ of dimension $\geq 2$ over a field $F$, such that $H$ is the multiplicative group of $F, T$ is the additive group of $V$, and the action $H \times T \rightarrow T$ is induced by the scalar multiplication $F \times V \rightarrow V$.

Proof. Let $G$ be the semi-direct product of $H$ by $T$ with respect to the given action: $G$ is the set $T \times H$ with multiplication

$$
(t, h)\left(t^{\prime}, h^{\prime}\right)=\left(t \cdot h t^{\prime}, h h^{\prime}\right)
$$

$H$ can be identified with the subgroup $\left\{\left(e_{T}, h\right) \mid h \in H\right\}$ of $G, T$ can be identified with the normal subgroup $\left\{\left(t, e_{H}\right) \mid t \in T\right\}$, and then the action of $H$ on $T$ is given by conjugation inside $G$. Note that:

(*) every element $g \in G$ is uniquely of the form $g=t h \quad(t \in T, h \in H)$.

In view of Remark (1.11) and the proof of Theorem 1, we can prove the implication $(1) \Rightarrow(2)$ by showing that the pair $G \supset H$ satisfies $(\mathrm{GH} 1),(\mathrm{GH} 2)^{*}$ and $(\mathrm{GH} 3)^{*}$; and that $T$ is the set of translations of $(G, H)$, i.e., a non-identity element $g \in G$ is in $T$ if and only if no conjugate of $g$ is in $H$.

For (GH1), choose $t, t^{\prime} \in T$ such that the orbits $\langle t\rangle,\left\langle t^{\prime}\right\rangle$, and $\left\langle e_{T}\right\rangle$ are distinct. Inside $G$, if $t^{\prime}$ were in $H t H$ we would have

$$
t^{\prime}=h t h^{\prime}=\left(h t h^{-1}\right)\left(h h^{\prime}\right) \quad\left(h, h^{\prime} \in H\right)
$$

whence, by $(*), t^{\prime}=h t h^{-1} \in\langle t\rangle$. So $t^{\prime} \notin H t H$, and the three double cosets $H t H, H t^{\prime} H$ and $H$ are distinct, proving (GH1).

For $(\mathrm{GH} 2)^{*}$, let $t \in T$ and consider an element $h \in H \cap t H t^{-1}$. We have

$$
e h=h=\left(t h_{1} t^{-1} h_{1}^{-1}\right) h_{1} \quad\left(h_{1} \in H\right)
$$

whence, by $(*), e=t h_{1} t^{-1} h_{1}^{-1}$ and $h=h_{1}$, so that $h t h^{-1}=t$. Thus if $h \in \bigcap_{t \in T} t H t^{-1}$, then $h t h^{-1}=t$ for all $t \in T$, and since $H$ acts faithfully on $T$, therefore $h=e$, proving (GH2)*

For $(\mathrm{GH} 3)^{*}$, note first that for any $t \in T$ we have, inside $G$,

$$
H\langle t\rangle=\langle t\rangle H
$$

as follows from the identities

$$
\begin{aligned}
& h_{1} h t h^{-1}=\left(h_{1} h t h^{-1} h_{1}^{-1}\right) h_{1} \\
& h t h^{-1} h_{1}=h_{1}\left(h_{1}^{-1} h t h^{-1} h_{1}\right) \quad\left(h, h_{1} \in H\right) .
\end{aligned}
$$

For any $g \in G$, writing $g=t h$, cf. $(*)$, we see then that

$$
H g H \cup H=H t H \cup H=H\langle t\rangle \cup H,
$$


and we deduce easily from (ii) in (1) and from (**) that $H g H \cup H$ is a subgroup of $G$, proving (GH3)*.

Finally, since the normal subgroup $T$ equals any of its conjugates in $G$, and since $T \cap H=$ $\{e\}$, it is clear that every $t \in T$ is a translation. Conversely, if $g=t h$ (cf. $(*))$ is a translation, then (1.7) implies that $g=t \in T$.

This completes the proof that $(1) \Rightarrow(2)$; and the implication $(2) \Rightarrow(1)$ is clear.

There is an obvious functor $\Gamma^{*}$ from the category $\mathbb{V}$ to the category whose objects are pairs $(T, H)$ satisfying the conditions in Theorem $1 \mathrm{~b}$, and whose arrows $(T, H) \rightarrow\left(T^{\prime}, H^{\prime}\right)$ are pairs $(\xi, \eta)$ where $\xi: T \rightarrow T^{\prime}$ is an injective group homomorphism and $\eta: H \rightarrow H^{\prime}$ is a group homomorphism such that

$$
\xi(h t)=\eta(h) \xi(t) \quad(h \in H, t \in T) .
$$

We leave it to the reader to verify that: $\Gamma^{*}$ is an equivalence of categories.

\section{Proof of Theorem 2.}

Proposition (2.1). Let $(V, F)$ and $\Gamma(V, F)=(G, H)$ be as in the Introduction. To each subspace $V^{\prime}$ of $V$ associate the subgroup $\Gamma_{0}\left(V^{\prime}\right)$ of $G$ given by

$$
\Gamma_{0}\left(V^{\prime}\right)=\left\{[a, v] \in G \mid 0 \neq a \in F, v \in V^{\prime}\right\}
$$

(1) $\Gamma_{0}$ is an inclusion preserving bijective map from the set of subspaces of $V$ onto the set of subgroups of $G$ containing $H$.

(2) For any element $0 \neq v \in V$ and any non-zero $c \in F$ we have

$$
\Gamma_{0}(F v)=(H[c, v] H) \cup H
$$

(3) For any two subspaces $V_{1} \subset V, V_{2} \subset V$, we have

$$
\Gamma_{0}\left(V_{1}+V_{2}\right)=\Gamma_{0}\left(V_{1}\right) \Gamma_{0}\left(V_{2}\right)=\Gamma_{0}\left(V_{2}\right) \Gamma_{0}\left(V_{1}\right)
$$

Proof. (1) First of all it is clear that $\Gamma_{0}\left(V^{\prime}\right)$ is a subgroup of $G$ containing $H$.

We show that $\Gamma_{0}$ is bijective by constructing an inverse map. For any subgroup $G^{\prime} \subset G$ with $G^{\prime} \supset H$ set

$$
\Theta_{0}\left(G^{\prime}\right)=\left\{v \in V \mid[1, v] \in G^{\prime}\right\}
$$

Since

$$
\left[1, v_{1}\right]\left[1, v_{2}\right]=\left[1, v_{1}+v_{2}\right]
$$

therefore $\Theta_{0}\left(G^{\prime}\right)$ is closed under addition; and since for non-zero $a \in F$ we have $[a, 0] \in H \subset$ $G^{\prime}$ and

$$
[a, 0][1, v][a, 0]^{-1}=[1, a v],
$$

therefore $\Theta_{0}\left(G^{\prime}\right)$ is closed under scalar multiplication; so $\Theta_{0}\left(G^{\prime}\right)$ is a subspace of $V$.

By definition

$$
v \in \Theta_{0} \Gamma_{0}\left(V^{\prime}\right) \Longleftrightarrow[1, v] \in \Gamma_{0}\left(V^{\prime}\right) \Longleftrightarrow v \in V^{\prime}
$$


i.e., $\Theta_{0} \Gamma_{0}\left(V^{\prime}\right)=V^{\prime}$.

Moreover, if $[1, v] \in G^{\prime}$ and $0 \neq a \in F$, then

$$
[a, v]=[1, v][a, 0] \in G^{\prime} ;
$$

and conversely if $[a, v] \in G^{\prime}$, then

$$
[1, v]=[a, v][a, 0]^{-1} \in G^{\prime} .
$$

It follows easily that $\Gamma_{0} \Theta_{0}\left(G^{\prime}\right)=G^{\prime}$, and (1) is proved.

(2) We have

$$
\begin{aligned}
\Gamma_{0}(F v) & =\{[a, b v] \mid 0 \neq a \in F, 0 \neq b \in F\} \cup\{[a, 0] \mid 0 \neq a \in F\} \\
& =\left\{[b, 0][c, v]\left[c^{-1} b^{-1} a, 0\right] \mid 0 \neq a \in F, 0 \neq b \in F\right\} \cup H \\
& =(H[c, v] H) \cup H .
\end{aligned}
$$

(3) The inclusions

$$
\Gamma_{0}\left(V_{2}\right) \Gamma_{0}\left(V_{1}\right) \subset \Gamma_{0}\left(V_{1}+V_{2}\right) \supset \Gamma_{0}\left(V_{1}\right) \Gamma_{0}\left(V_{2}\right)
$$

are obvious; and the opposite inclusions follow from the equalities

$$
\left[1, v_{2}\right]\left[a, v_{1}\right]=\left[a, v_{1}+v_{2}\right]=\left[1, v_{1}\right]\left[a, v_{2}\right] .
$$

Corollary (2.2). Given vectors $v_{0}, v_{1}, \ldots, v_{n} \in V$, and non-zero elements $c_{i} \in F(0 \leq i \leq$ $n)$, with $g_{i}=\left[c_{i}, v_{i}\right]$ we have that

$v_{0}$ is a linear combination of $v_{1}, v_{2}, \ldots, v_{n}$

$$
\Longleftrightarrow g_{0} \in\left(H g_{1} H \cup H\right)\left(H g_{2} H \cup H\right) \cdots\left(H g_{n} H \cup H\right) \text {. }
$$

Corollary (2.3). $\Gamma_{0}$ induces a one-one correspondence between the set of 1-dimensional subspaces of $V$ (i.e., the points of the projective space $P(V, F))$ and the set of double cosets $H g H \neq H$.

Corollary (2.4). Let $(\zeta, \theta):(V, F) \rightarrow\left(V^{\prime}, F^{\prime}\right)$ be a semi-linear map, and let

$$
f=\Gamma(\zeta, \theta): \Gamma(V, F)=(G, H) \rightarrow\left(G^{\prime}, H^{\prime}\right)=\Gamma\left(V^{\prime}, F^{\prime}\right) .
$$

Then $\zeta$ is linearly faithful $\Longleftrightarrow(0.3)$ holds.

Proof. Recall from the Introduction that $\zeta$ linearly faithful means that every subspace $V_{1} \subset V$ is of the form $\zeta^{-1}\left(V_{1}^{\prime}\right)$ for some subspace $V_{1}^{\prime} \subset V^{\prime}$. The conclusion follows easily from (1) in (2.1).

Let us show now that, as asserted in the Introduction, the functors $\Gamma$ and $\Theta$ induce pseudo-inverse equivalences between the categories $\mathbb{V}_{3} \subset \mathbb{V}$ and $\mathbb{G}_{3} \subset \mathbb{G}$.

It will suffice to show that (a): $\Gamma\left(\mathbb{V}_{3}\right) \subset \mathbb{G}_{3}$, and (b): $\Theta\left(\mathbb{G}_{3}\right) \subset \mathbb{V}_{3}$. Then any pair of isomorphisms $\Gamma \Theta \stackrel{\sim}{\longrightarrow}, \Theta \Gamma \stackrel{\sim}{\longrightarrow} \mathbf{1}$, will induce similar isomorphisms for the restrictions of 
$\Gamma$ and $\Theta$ to $\mathbb{V}_{3}$ and $\mathbb{G}_{3}$ respectively, because any isomorphism in $\mathbb{V}$ between objects of $\mathbb{V}_{3}$ is linearly faithful, and hence is an isomorphism in $\mathbb{V}_{3}$; and similarly any isomorphism in $\mathbb{G}$ between objects of $\mathbb{G}_{3}$ is an isomorphism in $\mathbb{G}_{3}$.

Assertion (a) follows easily for objects from (2.2) and for arrows from (2.4).

As for (b), let us first consider an object $(G, H) \in \mathbb{G}_{3}$, and set $\Theta(G, H)=(V, F)$. Then $(G, H)$ is isomorphic to $\Gamma(V, F)$ (in $\mathbb{G})$. By definition of $\mathbb{G}_{3}$,

$$
G \neq\left(H g_{1} H \cup H\right)\left(H g_{2} H \cup H\right) \quad \text { for all } g_{1}, g_{2} \in G,
$$

and it follows from (2.2) that $V$ is not spanned by two 1-dimensional subspaces, i.e., $\operatorname{dim} V_{3} \geq$ 3, i.e., $(V, F) \in \mathbb{V}_{3}$.

Let $f:(G, H) \rightarrow\left(G^{\prime}, H^{\prime}\right)$ be a map in $\mathbb{G}_{3}$, and let $(V, F)=\Theta(G, H),\left(V^{\prime}, F^{\prime}\right)=\Theta\left(G^{\prime}, H^{\prime}\right)$. Then the functorial isomorphism $\Gamma \Theta \stackrel{\sim}{\longrightarrow} \mathbf{1}$ gives a commutative diagram

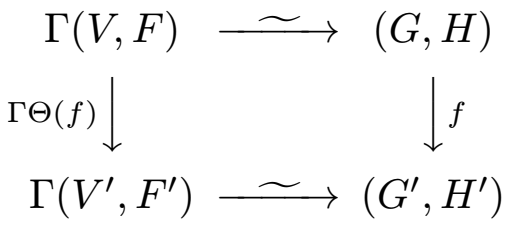

where the horizontal arrows are isomorphisms. Then $\Gamma \Theta(f)$ satisfies condition $(0.3)$ since $f$ does; and hence by $(2.4), \Theta(f)$ is linearly faithful. This completes the proof of (b).

Next let us note that $\Gamma$ and $\Theta$ respect the equivalence relations $\mathbf{R}$ and $\mathbf{R}^{*}$ defined in the Introduction. Indeed, if $(G, H)=\Gamma(V, F)$, and if $0 \neq a \in F$, so that $h=[a, 0] \in H$, then

$$
\Gamma\left(\zeta_{a}, \theta_{a}\right)=\gamma_{h} .
$$

Conversely, if $(V, F)=\Theta(G, H)$, then there is an $a \in F$ such that

$$
\Theta\left(\gamma_{h}\right)=\left(\zeta_{a}, \theta_{a}\right) .
$$

To see this, recall that there is a functorial isomorphism

$$
f:(G, H) \stackrel{\sim}{\longrightarrow} \Gamma \Theta(G, H)
$$

and hence $\Gamma \Theta\left(\gamma_{h}\right)=\gamma_{f(h)}$; but as we just saw, there is an $a$ such that $\Gamma\left(\zeta_{a}, \theta_{a}\right)=\gamma_{f(h)}$; and since the equivalence $\Gamma$ acts injectively on arrows, this $a$ is as desired.

The existence of the functor $\bar{\Theta}$ defined near the end of the Introduction follows easily, as does the existence of a unique functor $\bar{\Gamma}$ making the following diagram commute:

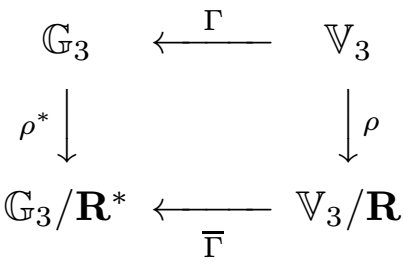

Finally, $\bar{\Theta}$ and $\bar{\Gamma}$ are pseudo-inverse equivalences. For example to get an isomorphism $\bar{\Gamma} \bar{\Theta} \stackrel{\sim}{\longrightarrow} \mathbf{1}_{\mathbb{G}_{3} / \mathbf{R}^{*}}$ we need for each $A=(G, H) \in \mathbb{G}_{3} / \mathbf{R}^{*}$ an isomorphism $\bar{\Gamma} \bar{\Theta}(A) \stackrel{\sim}{\longrightarrow}$. But $\bar{\Gamma} \bar{\Theta}(A)=\Gamma \Theta(A)$, and we have an isomorphism $f: \Gamma \Theta(A) \stackrel{\sim}{\longrightarrow} A$ in $\mathbb{G}_{3}$. The equivalence class of $f$ under $\mathbf{R}^{*}$ gives what we want.

Theorem 2 should now be clear. 


\section{REFERENCES}

E. Artin, Geometric Algebra, Interscience, New York, 1957.

J. Lipman, Definition of affine geometry by a group of transformations, Canad. Math. Bull. 4 (1961), 265278.

Department of Mathematics, Purdue University, W. Lafayette IN 47907 